\title{
SOME EXTENSIONS OF THE BROCK-CARLITZ IDENTITY
}

\author{
M. E. COHEN AND H. S. SUN
}

\begin{abstract}
The paper deals with a number of generalizations of an identity first introduced by Brock, and subsequently investigated in some detail by Carlitz. The theorem presented in this paper not only extends the formulas of Carlitz, but also shows that a multi-dimensional function satisfies the identity. A result is also deduced regarding the positive nature of the recurrence relation.
\end{abstract}

I. Introduction. Brock proposed the identity

$$
H(m, n)-H(m-1, n)-H(m, n-1)=\left(\begin{array}{c}
m+n \\
n
\end{array}\right)^{2}
$$

for

$$
H(m, n)=\sum_{i=0}^{m} \sum_{j=0}^{n}\left(\begin{array}{c}
i+j \\
i
\end{array}\right)\left(\begin{array}{c}
m-i+j \\
m-i
\end{array}\right)\left(\begin{array}{c}
i+n-j \\
n-j
\end{array}\right)\left(\begin{array}{c}
m-i+n-j \\
m-i
\end{array}\right) .
$$

The identity was subsequently proved by Slepian [1] and a second combinatorial proof was given by Baer and Brock [2]. Over a period of time, Carlitz [3]-[10] investigated (1.1) in many different directions, including multiple cyclic sums, generating functions, and higher order identities. Interest in (1.1) is heightened by the fact that it is connected with generating functions involving the Jacobi polynomial (see Carlitz [8], also Singhal [12] and Cohen [11]).

One of the papers by Carlitz [7] treats as a main result the expression

$$
\begin{aligned}
& H(m, n \mid r, s) \\
& \quad=\sum_{i=0}^{\min (m, r)} \sum_{j=0}^{\min (n, s)}\left(\begin{array}{c}
i+j \\
i
\end{array}\right)\left(\begin{array}{c}
m-i+j \\
m-i
\end{array}\right)\left(\begin{array}{c}
i+n-j \\
n-j
\end{array}\right)\left(\begin{array}{c}
r-i+s-j \\
r-i
\end{array}\right)
\end{aligned}
$$

which is shown to satisfy the relation

$$
\begin{gathered}
H(m, n \mid r, s)-H(m-1, n \mid r-1, s)-H(m, n-1 \mid r, s-1) \\
=\left(\begin{array}{c}
m+n \\
m
\end{array}\right)\left(\begin{array}{c}
r+s \\
r
\end{array}\right)
\end{gathered}
$$

for $m \leqslant r$ or $n \leqslant s$. Clearly, by letting $m=r, n=s$, (1.1) presents itself.

A special case of our theorem in this paper yields an extension of (1.2).

Received by the editors July 7, 1978.

AMS (MOS) subject classifications (1970). Primary 05A19, 05A20; Secondary 05A15.

Key words and phrases. Combinatorial identities, multiple cyclic sums, recurrence relations. 
Defining

$$
\begin{aligned}
H^{\beta}(m, n \mid r, s)= & \sum_{j=0}^{\min (n, s)} \sum_{i=0}^{\min (m, r)}\left(\begin{array}{c}
i+j \\
i
\end{array}\right)\left(\begin{array}{c}
m-i+j \\
m-i
\end{array}\right) \\
& \cdot\left(\begin{array}{c}
\beta+n-j+i \\
n-j
\end{array}\right)\left(\begin{array}{c}
r+s-i-j \\
r-i
\end{array}\right)
\end{aligned}
$$

then

$$
\begin{gathered}
H^{\beta}(m, n \mid r, s)-H^{\beta}(m-1, n \mid r-1, s)-H^{\beta}(m, n-1 \mid r, s-1) \\
=\left(\begin{array}{c}
r+s \\
s
\end{array}\right)\left(\begin{array}{c}
\beta+m+n \\
\beta+m
\end{array}\right)
\end{gathered}
$$

provided $m \leqslant r$ or $n \leqslant s . \beta=0$ in (1.4) gives (1.2). In point of fact our theorem gives a more general expression for

$$
\begin{array}{r}
H^{\beta, \gamma}(m, n \mid r, s)=\sum_{j=0}^{\min (n, s)} \sum_{i=0}^{\min (m, r)}\left(\begin{array}{c}
\gamma+i+j \\
i
\end{array}\right)\left(\begin{array}{c}
m-i+j \\
m-i
\end{array}\right) \\
\cdot\left(\begin{array}{c}
\beta+n-j+i \\
n-j
\end{array}\right)\left(\begin{array}{c}
r+s-i-j \\
r-i
\end{array}\right) \\
H^{\beta, \gamma}(m, n \mid r, s)-H^{\beta, \gamma}(m-1, n \mid r-1, s)-H^{\beta, \gamma}(m, n-1 \mid r, s-1) \\
=\left(\begin{array}{c}
\beta+m+n \\
\beta+m
\end{array}\right) \sum_{k=0}^{\min (m, r)}\left(\begin{array}{c}
r+s-k \\
s
\end{array}\right)\left(\begin{array}{c}
\gamma+k-1 \\
k
\end{array}\right)
\end{array}
$$

provided $m \leqslant r$ or $n \leqslant s$. For the restriction $m \geqslant r$ and $n<s$, the summation on the right-hand side of (1.6) reduces to

$$
\begin{gathered}
H^{\beta, \gamma}(m, n \mid r, s)-H^{\beta, \gamma}(m-1, n \mid r-1, s)-H^{\beta, \gamma} H(m, n-1 \mid r, s-1) \\
=\left(\begin{array}{c}
\beta+m+n \\
\beta+m
\end{array}\right)\left(\begin{array}{c}
\gamma+r+s \\
\gamma+s
\end{array}\right), \quad m \geqslant r \text { and } n \leqslant s .
\end{gathered}
$$

We may also deduce another result of the form

$$
\begin{gathered}
H^{\alpha, \beta}(m, n \mid r, s)-H^{\alpha, \beta}(m-1, n \mid r-1, s)-H^{\alpha, \beta}(m, n-1 \mid r, s-1) \\
=\left(\begin{array}{c}
r+s \\
s
\end{array}\right) \sum_{k=0}^{m}\left(\begin{array}{c}
\alpha+k-1 \\
k
\end{array}\right)\left(\begin{array}{c}
\beta+m+n-k \\
\beta+m-k
\end{array}\right)
\end{gathered}
$$

provided $m \leqslant r$ or $n \leqslant s$, where

$$
\begin{aligned}
H^{\alpha, \beta}= & \sum_{j=0}^{\min (n, s)} \sum_{i=0}^{\min (m, r)}\left(\begin{array}{c}
i+j \\
i
\end{array}\right)\left(\begin{array}{c}
\alpha+m-i+j \\
m-i
\end{array}\right) \\
& \cdot\left(\begin{array}{c}
\beta+n-j+i \\
n-j
\end{array}\right)\left(\begin{array}{c}
r+s-i-j \\
r-i
\end{array}\right) .
\end{aligned}
$$

Evidently $\alpha=\beta=0$ in (1.8) and $\gamma=\beta=0$ in (1.6) will give the Carlitz expression in (1.2).

Now recently Carlitz [9] reconsidered his identity (1.2) and studied it for 
the case $m>r$ and $n>s$. In the process he derived, as a main result, the expression

$$
\begin{array}{r}
H(m, n \mid r, s)-H(m-1, n \mid r-1, s)-H(m, n-1 \mid r, s-1) \\
=\left(\begin{array}{c}
r+s \\
r
\end{array}\right) \sum_{i=0}^{s}\left(\begin{array}{c}
i+m-r-1 \\
i
\end{array}\right)\left(\begin{array}{c}
n+r-i \\
r
\end{array}\right) .
\end{array}
$$

A special case of our theorem gives

$$
\begin{gathered}
H^{\beta, \gamma}(m, n \mid r, s)-H^{\beta, \gamma}(m-1, n \mid r-1, s)-H^{\beta, \gamma}(m, n-1 \mid r, s-1) \\
=\left(\begin{array}{c}
\gamma+r+s \\
\gamma+s
\end{array}\right) \sum_{i=0}^{s}\left(\begin{array}{c}
i+m-r-1 \\
i
\end{array}\right)\left(\begin{array}{c}
\beta+r+n-i \\
\beta+r
\end{array}\right)
\end{gathered}
$$

for $m>r$ and $n>s$. Evidently $\beta=\gamma=0$ in (1.11) results in the formula (1.10).

The expressions (1.6) and (1.11) permit us to make the following statement:

$$
H^{\beta, \gamma}(m, n \mid r, s)-H^{\beta, \gamma}(m-1, n \mid r-1, s)-H^{\beta, \gamma}(m, n-1 \mid r, s-1)>0
$$

for $\beta, \gamma>0$ and $m, n, r, s$ nonnegative integers, where $H^{\beta, \gamma}$ is defined by (1.5). The special case $\beta=\gamma=0$ was first given as a theorem by Carlitz [9, equation (18)].

For $\beta$ a nonnegative integer, $\gamma$ real or complex, we deduce a new expression

$$
\begin{aligned}
H^{\beta, \gamma}(2 r+\beta & +1,2 s+1 \mid r, s)-H^{\beta, \gamma}(2 r+\beta, 2 s+1 \mid r-1, s) \\
& -H^{\beta, \gamma}(2 r+\beta+1,2 s \mid r, s-1) \\
= & \frac{1}{2}\left(\begin{array}{c}
2 \beta+2 r+2 s+2 \\
2 \beta+2 r+1
\end{array}\right)\left(\begin{array}{c}
\gamma+r+s \\
\gamma+s
\end{array}\right) .
\end{aligned}
$$

Again, for $\beta=\gamma=0$, the known Carlitz result [9, equation (21)] presents itself. Note the misprints in Carlitz [9, equations (20), (21)].

The above special cases are some one-dimensional examples of the theorem. As an illustration, we give one two-dimensional special case of our multi-dimensional expression. The two-dimensional analog of (1.12) is

$$
F(m, n \mid r, s)-F(m-1, n \mid r-1, s)-F(m, n-1 \mid r, s-1)>0
$$

for $2 b-1>0,2 c-1>0$, and $m, n, r, s$ nonnegative integers, where

$$
\begin{aligned}
F(m, n r, s)= & \sum_{s_{1}=0}^{s} \sum_{r_{1}=0}^{r} \sum_{m_{1}=0}^{m} \sum_{n_{1}=0}^{n} f\left(m_{1}, n_{1} \mid r_{1}, s_{1}\right) \\
& \cdot f\left(m-m_{1}, n-n_{1} \mid r-r_{1}, s-s_{1}\right)
\end{aligned}
$$


and

$$
\begin{aligned}
f(m, n \mid r, s)= & \sum_{j=0}^{\min (n, s)} \sum_{i=0}^{\min (m, r)}\left(\begin{array}{c}
c+i+j-1 \\
i
\end{array}\right)\left(\begin{array}{c}
j-\frac{1}{2} \\
j
\end{array}\right) \\
& \cdot\left(\begin{array}{c}
m-i+j-\frac{1}{2} \\
m-i
\end{array}\right)\left(\begin{array}{c}
b+n-j+i-1 \\
n-j
\end{array}\right) \\
& \cdot\left(\begin{array}{c}
r+s-i-j-\frac{1}{2} \\
r-i
\end{array}\right)\left(\begin{array}{c}
s-j-\frac{1}{2} \\
s-j
\end{array}\right) .
\end{aligned}
$$

The multi-dimensional theorem is presented and derived in the next section.

II. ThEOREM. For $a, b, c$ real or complex numbers, $m, n, r, s$ nonnegative integers, and $\sigma$ a positive integer

$$
\begin{gathered}
F(m, n \mid r, s)-F(m-1, n \mid r-1, s)-F(m, n-1 \mid r, s-1) \\
=G(m, n \mid r, s)-H(m, n \mid r, s),
\end{gathered}
$$

where

$$
\begin{gathered}
G=\sum_{k=0}^{\min (m, r)} \sum_{p=0}^{n}\left(\begin{array}{c}
a \sigma+n+m-p-k-1 \\
m-k
\end{array}\right)\left(\begin{array}{c}
r+s-k \\
s
\end{array}\right) \\
\cdot\left(\begin{array}{c}
c \sigma+k-2 \\
k
\end{array}\right)\left(\begin{array}{c}
b \sigma+k+p-2 \\
p
\end{array}\right), \\
H=\left(\begin{array}{c}
c \sigma-1+r+s \\
c \sigma-1+s
\end{array}\right) \sum_{l=0}^{n-s-1}\left(\begin{array}{c}
b \sigma-1+r+l \\
l
\end{array}\right)\left(\begin{array}{c}
a \sigma-2+m+n-r-l \\
m-r-1
\end{array}\right),
\end{gathered}
$$

where $H=0$ for $m \leqslant r$ or $n \leqslant s$,

$$
\begin{aligned}
& F(m, n \mid r, s)=\sum_{\substack{n_{1}, n_{2}, \ldots, n_{o} \\
n_{i}>0}} \sum_{\substack{m_{1}, \ldots, m_{o} \\
m_{i}>0}} \sum_{\substack{r_{1}, \ldots, r_{o} \\
r_{i}>0}} \\
& \sum_{\substack{s_{1}, \ldots, s_{o} \\
s_{i}>0 \\
s_{1}+\cdots+s_{o}=s}} \prod_{i=1}^{o} f\left(m_{i}, n_{i} \mid r_{i}, s_{i}\right), \\
& f(m, n \mid r, s)=\sum_{j=0}^{\min (n, s)} \sum_{i=0}^{\min (m, r)}\left(\begin{array}{c}
c+i+j-1 \\
i
\end{array}\right)\left(\begin{array}{c}
1 / \sigma+j-1 \\
j
\end{array}\right) \\
& \cdot\left(\begin{array}{c}
a+m-i+j-1 \\
m-i
\end{array}\right)\left(\begin{array}{c}
b+n-j+i-1 \\
n-j
\end{array}\right) \\
& \cdot\left(\begin{array}{c}
1 / \sigma+r+s-i-j-1 \\
r-i
\end{array}\right)\left(\begin{array}{c}
1 / \sigma+s-j-1 \\
s-j
\end{array}\right)
\end{aligned}
$$


and

$$
\left(\begin{array}{l}
a \\
b
\end{array}\right)=\frac{\Gamma(a+1)}{\Gamma(b+1) \Gamma(a-b+1)} \text {, quotient of gamma functions. }
$$

CoRollary 1. $F_{1}(m, n \mid r, s)$ is obtained by letting $a=1 / \sigma$ in (2.4) and (2.5). Then

$$
\begin{gathered}
F_{1}(m, n \mid r, s)-F_{1}(m-1, n \mid r-1, s)-F_{1}(m, n-1 \mid r, s-1) \\
=G_{1}(m, n \mid r, s)-H_{1}(m, n \mid r, s)
\end{gathered}
$$

where

$$
G_{1}=\left(\begin{array}{c}
b \sigma-1+m+n \\
n
\end{array}\right) \sum_{k=0}^{\min (m, r)}\left(\begin{array}{c}
r+s-k \\
s
\end{array}\right)\left(\begin{array}{c}
c \sigma+k-2 \\
k
\end{array}\right)
$$

and $H_{1}$ is obtained from (2.3) by letting $a=1 / \sigma$.

COROLlaRY 2. With the restrictions $m>r$ and $n>s$ in Corollary 1

$$
\begin{gathered}
F_{1}(m, n \mid r, s)-F_{1}(m-1, n \mid r-1, s)-F_{1}(m, n-1 \mid r, s-1) \\
=G_{2}(m, n \mid r, s)
\end{gathered}
$$

where

$$
G_{2}=\left(\begin{array}{c}
c \sigma-1+r+s \\
c \sigma-1+s
\end{array}\right) \sum_{l=0}^{s}\left(\begin{array}{c}
b \sigma-1+r+n-l \\
b \sigma-1+r
\end{array}\right)\left(\begin{array}{c}
m-r+l-1 \\
l
\end{array}\right)
$$

which is the combination of $G$ and $H$.

Corollary 3. $F_{3}(m, n \mid r, s)$ is obtained by letting $c=1 / \sigma$ in (2.4) and (2.5). Then

$$
\begin{gathered}
F_{3}(m, n \mid r, s)-F_{3}(m-1, n \mid r-1, s)-F_{3}(m, n-1 \mid r, s-1) \\
=G_{3}(m, n \mid r, s)-H_{3}(m, n \mid r, s)
\end{gathered}
$$

where

$$
G_{3}=\left(\begin{array}{c}
r+s \\
s
\end{array}\right) \sum_{p=0}^{n}\left(\begin{array}{c}
a+m+p-1 \\
a+p-1
\end{array}\right)\left(\begin{array}{c}
b+n-p-2 \\
n-p
\end{array}\right)
$$

and $H_{3}$ is defined in (2.3) with $c=1 / \sigma$.

Corollary 4. For bo $-1>0, c \sigma-1>0, m, n, r, s$ nonnegative integers

$$
F_{1}(m, n \mid r, s)-F_{1}(m-1, n \mid r-1, s)-F_{1}(m, n-1 \mid r, s-1)>0
$$

where $F_{1}$ is defined in Corollary 1. 
COROLlaRY 5. For bo -1 a nonnegative integer, c real or complex

$$
\begin{aligned}
F_{1}(2 r+b \sigma, 2 s & +1 \mid r, s)-F_{1}(2 r+b \sigma-1,2 s+1 \mid r-1, s) \\
& -F_{1}(2 r+b \sigma, 2 s \mid r, s-1) \\
= & \frac{1}{2}\left(\begin{array}{c}
2 b \sigma+2 r+2 s \\
2 b \sigma+2 r-1
\end{array}\right)\left(\begin{array}{c}
c \sigma+r+s-1 \\
c \sigma+s-1
\end{array}\right)
\end{aligned}
$$

where $F_{1}$ is defined in Corollary 1.

Proof of THE THEOREM. Now

$$
\begin{aligned}
& \sum_{m, n, r, s=0}^{\infty} x^{m} y^{n} u^{r} v^{s} f(m, n \mid r, s) \\
& =\sum_{m, n, r, s, i, j=0}^{\infty} \frac{x^{m} y^{n} u^{r} v^{s}(u x)^{i}(v y)^{j}(c+j)_{i}(1 / \sigma)_{j}(a+j)_{m}(b+i)_{n}(1 / \sigma)_{r+s}}{j ! i ! m ! n ! r ! s !} \\
& =\frac{(1-x)^{1 / \sigma-a}(1-y)^{c-b}(1-u x-y)^{1 / \sigma-c}}{(1-u-v)^{1 / \sigma}[(1-x)(1-y)-u x(1-x)-v y(1-y)]^{1 / \sigma}}
\end{aligned}
$$

(2.15) is derived from (2.14) by multiple series transformations, and (2.16) is subsequently deduced by simplifying the individual series.

(2.16) may be expressed as

$$
\begin{aligned}
&(1-x)^{1 / \sigma-a}(1-y)^{c-b}(1-u x-y)^{1 / \sigma-c} \\
& \quad\left\{\frac{1}{(1-u x-v y)(1-x-y)}\right. \\
&\left.\quad \cdot\left[\frac{1}{1-u-v}-\frac{x y}{(1-x)(1-y)-u x(1-x)-v y(1-y)}\right]\right\}^{1 / \sigma} .
\end{aligned}
$$

Equating (2.14) with (2.17), and raising both sides to the power $\sigma$, one has

$$
\begin{aligned}
& (1-x)^{1-a \sigma}(1-y)^{c \sigma-b o}(1-u x-y)^{1-c \sigma} \\
& \cdot\left\{\frac{1}{1-x-y}\right. \\
& \left.\quad \cdot\left[\frac{1}{1-u-v}-\frac{x y}{(1-x)(1-y)-u x(1-x)-v y(1-y)}\right]\right\} \\
& . \quad(1-u x-v y)\left\{\sum_{m, n, r, s=0}^{\infty} x^{m} y^{n} u^{r} v^{s} f(m, n \mid r, s)\right\}^{\circ} .
\end{aligned}
$$


(2.18) is treated in two parts. Consider

$$
\begin{aligned}
& \frac{(1-x)^{1-a \sigma}(1-y)^{1-b o}\{1-u x /(1-y)\}^{1-c o}}{(1-x-y)(1-u-v)} \\
& =\sum_{i, j, k, m_{1}, m_{2}, n_{1}, n_{2}=0}^{\infty} \frac{x^{m_{1}+k+y^{m_{2}+j} u^{n_{1}+m_{1}} \omega^{n_{2}}(c \sigma-1)_{m_{1}}(i+j) !\left(n_{1}+n_{2}\right) !}}{m_{1} ! i ! j ! n_{1} ! n_{2} !} \\
& \frac{(a \sigma-1)_{k}\left(m_{1}+b \sigma-1\right)_{m_{2}}}{k ! m_{2} !} \\
& =\sum_{m, n, r, s=0}^{\infty} x^{m^{n}} y^{n} u^{r} v^{s} \sum_{m_{1}, m_{2}, k=0}^{\infty} \frac{(c \sigma-1)_{m_{1}}\left(m+n-k-m_{1}-m_{2}\right) !}{m_{1} !\left(m-k-m_{1}\right) !\left(n-m_{2}\right) !} \\
& \frac{\left(r+s-m_{1}\right) !(a \sigma-1)_{k}\left(m_{1}+b \sigma-1\right)_{m_{2}}}{\left(r-m_{1}\right) ! s ! k ! m_{2} !} \\
& =\sum_{m, n, r, s=0}^{\infty} x^{m} y^{n} u^{r} v^{s} \sum_{p=0}^{n} \sum_{k=0}^{\min (m, r)}\left(\begin{array}{c}
a \sigma+n+m-p-k-1 \\
m-k
\end{array}\right) \\
& \cdot\left(\begin{array}{c}
r+s-k \\
s
\end{array}\right)\left(\begin{array}{c}
c \sigma+k-2 \\
k
\end{array}\right)\left(\begin{array}{c}
b \sigma+k+p-2 \\
p
\end{array}\right) \text {. }
\end{aligned}
$$

(2.21) is the expansion of (2.20), (2.22) involves series transformations, and (2.23) is deduced by reducing one of the series. This completes the expansion of the first part of (2.18).

The second part of (2.18)

$$
\begin{aligned}
& \frac{(1-x)^{1-a \sigma}(1-y)^{c \sigma-b o}(1-u x-y)^{1-c \sigma}}{1-x-y} \\
& \cdot\left\{\frac{x y}{(1-x)(1-y)-u x(1-x)-v y(1-y)}\right.
\end{aligned}
$$

may be expressed as

$$
\begin{aligned}
x y \sum_{n_{1}, n_{2}, i, j, p=0}^{\infty} \frac{\left(n_{1}+n_{2}\right) !(i+j) !(c \sigma-1)_{p}(1-x)^{-a \sigma-j}(1-y)^{-b \sigma-i-p}}{n_{1} ! n_{2} ! i ! j ! p !} & \cdot x^{n_{1}+i+p_{y} n_{2}+j} u^{i+p} v^{j} \\
=x y & \sum_{m, n, r, s=0}^{\infty} x^{m} y^{n} u^{r} v^{s} \sum_{l=0}^{n-s-1} \\
& \frac{(b \sigma+r)_{l}(-1)^{m}(1-c \sigma-r-s)_{r}(-a \sigma-m-n+r+l)_{m-r}}{l !(m-r) ! r !}
\end{aligned}
$$


(2.18) is now equal to the difference between (2.23) and (2.26). Equating (2.19) with this difference, and comparing coefficients, results in the identity (2.1).

\section{REFERENCES}

1. Problem 60-2, SIAM Rev. 4 (1962), 396-398.

2. R. M. Baer and P. Brock, Natural sorting, J. Soc. Indust. Appl. Math. 10 (1962), 284-304.

3. L. Carlitz, A binomial identity arising from a sorting problem, SIAM Rev. 6 (1961), 20-30.

4. __ Some multiple sums and binomial identities, SIAM J. Appl. Math. 13 (1965), $469-486$.

5. __ Multiple sums and generating functions, Collect. Math. 17 (1965), 3-18.

6. ___ Some binomial coefficient identities, Fibonacci Quart. 4 (1966), 323-331.

7. __ A binomial identity, SIAM Rev. 9 (1967), 229-231.

8. Some generating functions and a binomial identity, Duke Math. J. 35 (1968), 541-548.

9. __ Note on a binomial identity, SIAM J. Math. Anal. 6 (1975), 901-905.

10. Multiple binomial and power sums (private communication).

11. M. E. Cohen, Some extensions of Carlitz on cyclic sums and generating functions, J. Math. Anal. Appl. 60 (1977), 493-501.

12. J. P. Singhal, A generating function and a binomial identity, Duke Math. J. 39 (1972), 25-30.

Department of Mathematics, California State University, Fresno, California 93740 